\title{
DERIVAÇÃO DA FREQUÊNCIA INERCIAL EFETIVA DIRETAMENTE DE FORMULAÇÕES DESCRITIVAS DA TURBULÊNCIA
}

\author{
Arcilan Assireu ${ }^{1, *}$, João Antônio Lorenzzetti ${ }^{2}$, Cláudio Pellegrini $^{3}$ \\ ${ }^{1}$ UNIFEI, Itajubá, MG, ${ }^{2}$ INPE, São José dos Campos, SP, ${ }^{3}$ UFSJ, São João del-Rei, MG \\ *assireu@gmail.com
}

\section{RESUMO}

NIILER (1969) e MOERS (1975) introduziram o conceito da Frequência Inercial Efetiva (FIE), derivada a partir de considerações físicas associadas com escalas espaciais e de gradiente horizontal de velocidade. Neste trabalho, é apresentada uma derivação formal para a FIE e é mostrado que, para escoamentos turbulentos, a modificação da frequência inercial ocorre em uma forma muito mais geral do que inicialmente previsto pelos supra citados autores.

\begin{abstract}
NIILER (1969) and MOOERS (1975) introduced the concept of a modified inertial frequency, named Effective Inertial Frequency (EIF), based on physical considerations associated with frontal space and velocity scales. We present a formal derivation of EIF and show that for predominantly turbulent flow, the modification of the inertial frequency occurs in a much more general way than predicted by the space and velocity scale approximation.
\end{abstract}

\section{INTRODUÇÃO}

Evidências teóricas e observacionais suportam a ideia de que, quando o componente vertical da vorticidade relativa $(\zeta$ ) representa uma fração significativa da componente vertical da vorticidade planetária $(f)$, é mais apropriado o uso da frequência inercial efetiva (FIE). Frentes, correntes, jatos e vórtices podem influenciar os processos quase inerciais via interação não linear com o escoamento médio (MOOERS, 1975, KUNZE, 1985, WELLER et al. 1982, POULAIN et al., 1992, YOUNG and BEN JELLOUL, 1997, VAN MEURS, 1997, SHERMAN, 2005, SOBARZO et al. 2007). A relação formal para esta interação foi proposta por 
KUNZE (1985) ( FIE $\approx f+\zeta / 2)$ a qual foi derivada sob a hipótese de uma estratificação contínua e sob argumentos de escala que, na maioria das vezes, torna restritiva a teoria.

É apresentada neste trabalho uma derivação da FIE diretamente na Equação de NavierStokes (ENS) onde é mostrado que o FIE pode ser generalizado ao se assumir que a maioria dos escoamentos geofísicos apresentam comportamento turbulento.

\section{A FREQUÊNCIA INERCIAL EM ESCOAMENTOS TURBULENTOS}

A discussão que segue é baseada em TENNEKES e LUMLEY (1972). A principal diferença consiste do fato de que é considerada, neste trabalho, a equação do movimento, levando em conta um sistema de coordenadas que gira com velocidade angular $\Omega_{k}$ (associada à rotação da Terra) em oposição ao um sistema de coordenadas não rotacional. A vorticidade relativa $\omega$ é dada como

$$
\omega_{i}=\varepsilon_{i j k} \frac{\partial u_{k}}{\partial x_{j}}
$$

em que $\partial u_{k} / \partial x_{j}$ é a taxa de deformação e $\varepsilon_{i j k}$ é o tensor de permutação. A taxa de deformação é expressa como a soma dos componentes simétricos $\left(s_{i j}\right)$ e antissimétricos $\left(r_{i j}\right)$. Nós necessitamos considerar somente o tensor antissimétrico dado por:

$$
r_{i j}=\frac{1}{2}\left(\frac{\partial u_{i}}{\partial x_{j}}-\frac{\partial u_{j}}{\partial x_{i}}\right)
$$

Alguns dos termos da ENS podem ser reescritos em termos de vorticidade a partir das expressões previamente definidas. Reescrevendo o termo de advecção $u_{j} \partial u_{i} / \partial x_{j}$ na ENS na forma de um tensor de gradiente, obtém-se: 


$$
\frac{\partial u_{i}}{\partial t}=-\frac{1}{\rho} \frac{\partial p}{\partial x_{i}}-\frac{\partial}{\partial x_{j}}\left(u_{i} u_{j}\right)+v \frac{\partial^{2} u_{i}}{\partial x_{j} \partial x_{j}}+2 \varepsilon_{i j k} u_{j} \Omega_{k}
$$

Aplicando-se em 3 a equação da continuidade e reescrevendo

a contribuição da viscosidade também pode ser reescrita em (3) chega-se a:

$$
\frac{\partial u_{i}}{\partial t}=-\frac{1}{\rho} \frac{\partial p}{\partial x_{i}}-\frac{1}{2} \frac{\partial}{\partial x_{i}}\left(u_{j} u_{j}\right)+\varepsilon_{i j k} u_{j} \omega_{k}-v \varepsilon_{i j k} \frac{\partial \omega_{k}}{\partial x_{j}}+2 \varepsilon_{i j k} u_{j} \Omega_{k}
$$

Em um escoamento desprovido de rotação tem-se que $\omega_{k}=0$ e a Eq. (4) torna-se:

$$
\frac{\partial u_{i}}{\partial t}=-\frac{1}{\rho} \frac{\partial p}{\partial x_{i}}-\frac{1}{2} \frac{\partial}{\partial x_{i}}\left(u_{j} u_{j}\right)+2 \varepsilon_{i j k} u_{j} \Omega_{k} .
$$

Para escoamentos turbulentos, a simplificação acima não se aplica. O termo $\varepsilon_{i j k} u_{j} \omega_{k}$ é crucial para a teoria da turbulência. (TENNEKES and LUMLEY, 1972). Note que este termo é análogo a aceleração de Coriolis $2 \varepsilon_{i j k} u_{j} \Omega_{k}$. O fator 2 é ausente na vorticidade porque esta é duas vezes a velocidade angular do escoamento. Portanto, a partir da combinação destes dois termos, vê-se que o "novo" termo de Coriolis para escoamentos turbulentos fica:

$$
\varepsilon_{i j k}\left(\omega_{k}+2 \Omega_{k}\right) u_{j}
$$

Aplicando $\omega_{k}=2 \Omega_{k f}$, onde $\Omega_{k f}$ é a velocidade angular do fluido, em (6) e fazendo-se $\omega_{k}=\zeta=\partial u / \partial x-\partial v / \partial y$ em que $\zeta$ é a vorticidade relativa, conclui-se que o termo $f=2 \Omega \sin \phi$ nesta nova formulação é equivalente a: 


$$
f+\zeta / 2=f_{*}
$$

onde $f_{*}$, a frequência inercial efetiva (FIE), é a vorticidade planetária relacionada a frequência de Coriolis perturbada pela metade da vorticidade relativa do fluido.

\section{CONCLUSÃO}

A Equação (12), embora formalmente lembre a vorticidade absoluta (PEDLOSKY, 1979), não surgiu da equação da vorticidade e, dinamicamente, a sua conotação é diferente da vorticidade absoluta. A obtenção de $f_{*}$, a partir da Eq. (12), diferentemente dos autores citados ao longo deste trabalho não é baseada em considerações de escala, tendo surgido diretamente dos termos inerciais da ENS. Isto repercute em uma generalização para a FIE.

\section{AGRADECIMENTOS}

À FAPEMIG e ao CNPq pelo apoio.

\section{REFERÊNCIAS BIBLIOGRÁFICAS}

MOOERS, C. N. K. Several effects of a baroclinic current on the cross-stream propagation of inertial-waves. Geophysical Fluid Dynamics, 6, 245-275, 1975.

NIILER, P. P. On the Ekman divergence in an Oceanic jet. Journal of Geophysical Research., 74, 7048-7052, 1969.

PEDLOSKY, J. Geophysical Fluid Dynamics, Springer-Verlag, New York., 31, 624pp, 1979.

TENNEKES, H., and J. L. LUMLEY. A first course in Turbulence, The MIT Press, 300pp, 1972. 